1 Hacettepe Journal of Mathematics and Statistics

Volume 46 (6) (2017), 1035-1042

\title{
Soft congruence relation over lattice
}

\author{
Susanta Bera*, Sankar Kumar Roy ${ }^{\dagger, \ddagger}$, Faruk Karaaslan $^{\S}$ and Naim Çağman ${ }^{\star}$
}

\begin{abstract}
In this paper, we first describe soft congruence relation over a lattice. We then define the concepts of complete soft congruence relation. Besides this, the concepts of upper and lower approximations of a subset in a lattice are depicted based on this soft congruence relation. We then give their related properties with examples to investigate their characterizations.
\end{abstract}

Keywords: Rough sets, Soft sets, Lattices, Soft congruence relations.

2000 AMS Classification: 03G10,06B10.

Received : 20.09.2016 Accepted : 18.01.2017 Doi : 10.15672/HJMS.2017.436

\section{Introduction}

In our complex world, there are many situations occur, where we cannot use traditional methods to solve problems in economics, engineering, environmental science, social science, medical science etc. because of various types of uncertainties present in these problems. Probability theory, fuzzy set theory [31], rough set theory [18] are novel mathematical tools to solve real world problems including uncertainty.

Pawlak initiated rough set theory [19] to study incomplete knowledge which are found in the economics, engineering, and environmental science, computer science, and many other fields. Pawlak's rough set approximations are defined by means of an equivalence relation namely indiscernibility relation. Rough set is a pair of two crisp sets called lower and upper approximations and viewed as the sets of elements which certainly and possibly belong to the set. Pawlak [18] introduced the theory of rough set as an extension of set theory for the study of incomplete information. The relationship between rough sets and

*Department of Applied Mathematics with Oceanology and Computer Programming Vidyasagar University, Midnapore-721102, West Bengal, India, Email: bera.bapi@gmail.com

${ }^{\dagger}$ Department of Applied Mathematics with Oceanology and Computer Programming

Vidyasagar University, Midnapore-721102, West Bengal, India, Email: sankroy2006@gmail.com

$\ddagger$ Corresponding Author, Email: sankroy2006@gmail.com

${ }^{\S}$ Department of Mathematics, Çankiri Karatekin University, 18100 Çankiri, Turkey, Email: fkaraaslan@karatekin.edu.tr

${ }^{\top}$ Department of Mathematics, University of Gaziosmanpasa, 60100 Tokat, Turkey, Email: naim. cagman@gop.edu.tr 
algebraic systems (lattice) has been discussed by many researchers. For example, Iwinski [10] defined rough lattice and rough order without using any indiscernibility concept of a rough set. Järvinen [11] established lattice structure on rough sets which played an important role in rough set and Pawlak's information system. Xiao and Zhang [28] described the notion of rough prime ideals and rough fuzzy prime ideals in a semigroup. Rana and Roy [29] presented a unique approach to form lattice structure by choice function in rough set. Rasouli and Davvaz [30] gave the relationship between rough sets and ring theory, and introduced the concept of rough ideal with respect to an ideal of ring, which is an extended notion of ideal in a ring. Estaji et al. [8] studied the concept of upper and lower rough ideals in a lattice.

Molodtsov [14] defined soft set theory which is a new approach for dealing with vagueness and uncertainty in the real life problems. A soft set, in fact, is a tuple which associates with a set of parameters and a mapping from the parameter set into the power set of an universe set. Maji et al. [15] studied on the theory of soft sets initiated by Molodtsov, and developed several basic notions of soft set theory. Ali et al. [1] introduced the notion of restricted union, restricted intersection, restricted difference, and extended intersection between two soft sets. They established the notion of complement in soft set, and also proved that De Morgan's laws hold in soft set theory. Furthermore, Babitha and Sunil [2] gave definition of soft set relation based on cartesian product of two soft sets. After that, Babitha and Sunil [3] defined the partially ordered soft set by introducing ordering on soft sets, equivalent soft set relation, partition, composition and function. Park et al. [17] focused the discussion on equivalence relation, and they established that complete lattice is defined on the poset of equivalence soft set relations under a soft set. Applications of soft set theory in various field have been found in ([6], [7], [9], [12], [16], [20], [21]).

Lattice is a partially ordered set in which supremum and infimum exist for every pair of elements in the set. Algebraic structure (lattice) of rough set and soft set and their hybridization is an interesting topic to the researchers ([13], [19], [22]-[27]). Soft set theory has potential applications in many different fields due to its no necessity to describe the membership function. As a result, this makes that the soft set theory is so simple and popular in applications of various areas ([4], [5], [7], [12]).

In this paper, we describe the soft binary relation as well as soft congruence relation over lattice. We obtain some important properties of soft binary relation considering lattice as a universal set. Moreover, based on the ideas of congruence relation, we define soft congruence relation on lattice. Beside this, we approximate the subset of a lattice under soft congruence approximation space, and investigate the characteristic of ideal of the lattice under this soft congruence approximation space.

The remainder of the paper is designed as follows. In Section 2, we recall some basic concepts with respect to soft sets. Section 3 explores the soft binary relations over lattice including the various properties related to the soft binary relation. Approximation of a subset of a lattice under soft congruence relation is initiated in Section 4. Finally, conclusions and further study are discussed in Section 5.

\section{Preliminaries}

In this section, we present some basic definitions and results of soft set theory which are the most useful in the sequel.

Let $U$ be an initial universe set and let $E$ be a set of parameters which are usually initial attributes, characteristic, properties of object in $U$. Also let $P(U)$ denote the power set of $U$ and $A \subseteq E$. 
2.1. Definition. [15] A pair $(F, A)$ is called a soft set over $U$, where $F$ is a set valued function defined by $F: A \rightarrow P(U)$.

In other words, a soft set over $U$ is a parameterized family of subsets of $U$. That is, a soft set $(F, A)$ over $U$ can be presented as $(F, A)=\{(e, F(e)), e \in A\}$.

2.2. Example. Let $(F, A)$ describe the group of students interested in different subjects. Suppose there are four students in the universe $U$ which is given by $U=\left\{u_{1}, u_{2}, u_{3}, u_{4}\right\}$ and the attribute set $A=\left\{e_{1}, e_{2}, e_{3}\right\}$ where $e_{1}$ stands for Mathematics, $e_{2}$ stands for Science, $e_{3}$ stands for English. Suppose

$$
F\left(e_{1}\right)=\left\{u_{1}, u_{2}, u_{3}\right\}, F\left(e_{2}\right)=\left\{u_{1}, u_{2}\right\}, F\left(e_{3}\right)=\left\{u_{2}, u_{3}, u_{4}\right\}
$$

Thus the soft set over $U$ is given by

$$
(F, A)=\left\{\left(e_{1},\left\{u_{1}, u_{2}, u_{3}\right\}\right),\left(e_{2},\left\{u_{1}, u_{2}\right\}\right),\left(e_{3},\left\{u_{2}, u_{3}, u_{4}\right\}\right)\right\} .
$$

2.3. Definition. [15] Let $(F, A)$ and $(G, B)$ be two soft sets over $U .(G, B)$ is said to be a soft subset of $(F, A)$, if the following conditions are satisfied;

(1) $B \subseteq A$,

(2) $F(a)=G(a), \forall a \in B$.

We denote $(G, B) \sqsubseteq(F, A)$ where $\sqsubseteq$ stands for subset over a soft set. Two soft sets $(F, A)$ and $(G, B)$ over a common universe $U$ are said to be equal if $(G, B) \sqsubseteq(F, A)$ and $(F, A) \sqsubseteq(G, B)$.

2.4. Definition. [15] A soft set $(F, A)$ over $U$ is said to be a null soft set and is denoted by $F_{\phi}$ if $\forall a \in A, F(a)=\phi$ (null set).

2.5. Definition. [15] A soft set $(F, A)$ over $U$ is said to be an absolute soft set and is denoted by $F_{U}$ if $\forall a \in A, F(a)=U$.

2.6. Definition. [15] Union of two soft sets $(F, A)$ and $(G, B)$ over a common universe $U$ is a soft set $(H, C)$ where $C=A \cup B$ and $\forall a \in C, H$ is defined as follows:

$$
H(a)= \begin{cases}F(a), & \text { if } a \in A-B, \\ G(a), & \text { if } a \in B-A, \\ F(a) \cup G(a), & \text { if } a \in A \cap B .\end{cases}
$$

We write $(H, C)=(F, A) \sqcup(G, B)$ where the symbol ' $\sqcup$ ' stands for union between two soft sets.

2.7. Definition. [1] Intersection of two soft sets $(\gamma, A)$ and $(\delta, B)$ over a common universe $U$ is a soft set $(Y, D)$ where $D=A \cup B$, and $\forall a \in D$, and $Y$ is described as follows:

$$
Y(a)= \begin{cases}\gamma(a), & \text { if } a \in A-B \\ \delta(a), & \text { if } a \in B-A \\ \gamma(a) \cap \delta(a), & \text { if } a \in A \cap B .\end{cases}
$$

We write $(Y, D)=(\gamma, A) \sqcap(\delta, B)$ where the notation ' $\square$ ' stands for intersection between two soft sets.

2.8. Definition. [7] Let $(F, A)$ and $(G, B)$ be two soft sets over $U$. Then $A N D$-product of $(F, A)$ and $(G, B)$ is defined as follows:

$$
(H, C)=(F, A) \wedge(G, B)
$$

where $C=A \times B$ and $H(a, b)=F(a) \cap G(b)$ for all $(a, b) \in A \times B$. 


\section{Soft congruence relation on lattice}

In this section, we introduce the concept of soft congruence relation over lattice and thereafter we present their related properties.

Let us first, we discuss some basic notions of lattice theory. Suppose that $(L, \leq)$ is a partially order set. For all $a, b \in L$ if $a \vee b$ and $a \wedge b$ exist, then $L$ is called a lattice, where $a \vee b$ and $a \wedge b$ are supremum and infimum of $\{a, b\}$ respectively. Let $L$ be a lattice and $A \subseteq L$. Then $A$ is called a sublattice of $L$ if $a \in A ; b \in A$ imply $a \vee b \in A$ and $a \wedge b \in A$. $A$ is called an ideal if

(1) $a \in A$ and $b \in A$ imply $a \vee b \in A$,

(2) $a \in L, b \in A$ imply $a \wedge b \in A$.

3.1. Definition. An equivalence relation $\rho$ on a lattice $L$ is called a congruence relation if $a \rho b$ and $c \rho d$ hold and imply that $(a \wedge c) \rho(b \wedge d)$ and $(a \vee c) \rho(b \vee d)$ together hold.

Since $\rho$ is an equivalence relation on $L$, then $\rho$ would partition $L$ in equivalence classes where for any $a \in L$, equivalence class of $a$ is given as

$$
[a]_{\rho}=\{x \in L: x \rho a\}
$$

3.2. Definition. [9] Let $(\rho, A)$ be a soft set over $L \times L$, where $\rho$ is a set valued function defined by $\rho: A \rightarrow P(L \times L)$. Then $(\rho, A)$ is called a soft binary relation over $L$.

3.3. Definition. Let $(\rho, A)$ be soft binary relation over $L$. $(\rho, A)$ is called a soft equivalence relation over $L$ if each $\rho(e)[\neq \phi], e \in A$ is an equivalence relation on $L$.

3.4. Definition. A soft equivalence relation $(\rho, A)$ over $L$ is called a soft congruence relation over $L$ if each non null $\rho(e), e \in A$ is a congruence relation on $L$.

3.5. Example. Let $L=\{a, b, c, d\}$ be a lattice. The partial order on $L$ is defined as shown in Figure 1 and $A=\{\alpha, \beta\}$. Let us consider a set valued function $\rho: A \rightarrow P(L \times L)$ which is given by

$$
\rho(\alpha)=\{(a, a),(b, b),(c, c),(d, d),(a, c),(c, a),(b, d),(d, b)\}
$$

and

$$
\begin{aligned}
\rho(\beta)= & \{(a, a),(b, b),(c, c),(d, d),(a, c),(c, a),(b, c),(c, b),(a, b),(b, a),(d, c), \\
& (c, d),(a, d),(d, a),(b, d),(d, b)\}
\end{aligned}
$$

Then $(\rho, A)$ is a soft congruence relation on $L$

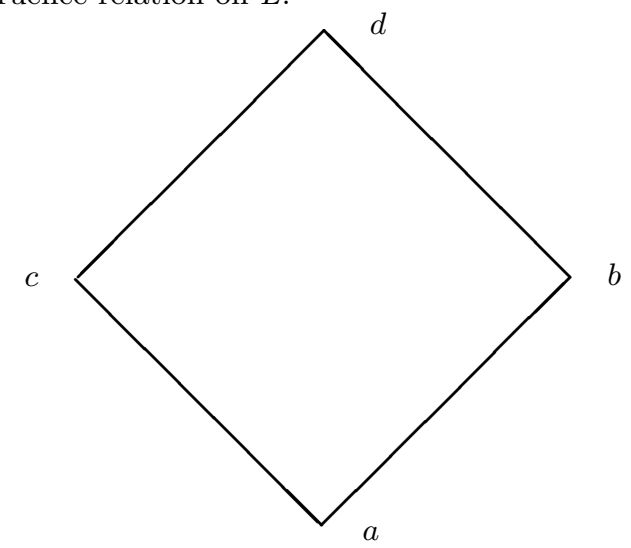

Figure 1: Lattice 
3.6. Proposition. Let $(F, A)$ and $(G, B)$ be soft congruence relations over $L$. Then $(H, C)=(F, A) \sqcup(G, B)$ is soft congruence relation over $L$ if $F(e) \subseteq G(e)$ or $G(e) \subseteq F(e)$ for all $e \in A \cup B$.

Proof. From Definition 2.6, we know that

$$
H(e)= \begin{cases}F(e), & \text { if } e \in A-B, \\ G(e), & \text { if } e \in B-A, \\ F(e) \cup G(e), & \text { if } e \in A \cap B\end{cases}
$$

for all $e \in C$. Suppose that $e \in A-B$. Then $H(e)=F(e)$. Since $F(e)$ is a congruence relation on $L, H(e)$ is a congruence relation on $L$. Suppose that $e \in B-A$. Then $H(e)=G(e)$. Since $G(e)$ is a congruence relation on $L, H(e)$ is a congruence relation on $L$. Let $e \in A \cap B$. Since $F(e) \subseteq G(e)$ or $G(e) \subseteq F(e), H(e)=F(e) \cup G(e)=F(e)$ or $H(e)=F(e) \cup G(e)=G(e)$. Since $F(e)$ and $G(e)$ are congruence relations, $H(e)$ is congruence relation. Hence the proof is completed.

3.7. Proposition. Let $(F, A)$ and $(G, B)$ be two soft congruence relations over $L$. Then, $(H, C)=(F, A) \sqcap(G, B)$ is soft congruence relation over $L$.

Proof. From Definition 2.7, we know that

$$
H(e)= \begin{cases}F(e), & \text { if } e \in A-B \\ G(e), & \text { if } e \in B-A \\ F(e) \cap G(e), & \text { if } e \in A \cap B\end{cases}
$$

for all $e \in C$. Suppose that $e \in A-B$. Then $H(e)=F(e)$. Since $F(e)$ is a congruence relation on $L, F(e)$ is a congruence relation on $L$. Suppose that $e \in B-A$. Then $H(e)=G(e)$. Since $G(e)$ is a congruence relation on $L, G(e)$ is a congruence relation on $L$. Let $e \in A \cap B$, then $H(e)=F(e) \cap G(e)$. Since intersection of two congruence relations is a congruence relation, $H(e)$ is a congruence relation over $L$. Hence $(F, A) \sqcap(G, B)$ is congruence relation.

\section{Approximations under soft congruence relation}

Let $(\rho, A)$ be a soft congruence relation on $L$. Then each $\rho(e), e \in A$ is a congruence relation over $L$. Let $\psi=\bigcap_{e \in A} \rho(e)$. Then $\psi$ is a congruence relation on $L$. The equivalence class of $x \in L$ under this congruence relation is described as $[x]_{\psi}=\{y \in L:(x, y) \in \psi\}$.

We define the pair $(L, \psi)$ as soft congruence approximation space.

4.1. Definition. Let $(L, \psi)$ be a soft congruence approximation space and $X$ be a nonempty subset of $L$. Then the lower and upper approximations of $X$ are defined as:

(1) $\psi_{\star}(X)=\left\{y \in L:[y]_{\psi} \subseteq X\right\}$,

(2) $\psi^{\star}(X)=\left\{y \in L:[y]_{\psi} \cap X \neq \phi\right\}$.

If $\psi_{\star}(X)=\psi^{\star}(X)$ then $X$ is called definable otherwise $X$ is called rough.

4.2. Example. Considering Example 3.5, then

$$
\psi=\cap_{\alpha \in A} \rho(\alpha)=\{(a, a),(b, b),(c, c),(d, d),(a, c),(c, a),(b, d),(d, b)\}
$$

is a congruence relation on $L$. The congruence classes are given by $\{a, c\},\{b, d\}$. Let $X=\{a, c, d\}$, then in the soft congruence approximation space $(L, \psi), \psi_{\star}(X)=\{a, c\}$ and $\psi^{\star}(X)=\{a, b, c, d\}$. Clearly $X$ is rough.

4.3. Definition. Let $\psi$ be a congruence relation on $L$ and $S$ be a non-empty subset of $L$. $S$ is called an upper rough ideal (sublattice) of $L$ if $\psi^{\star}(S)$ is an ideal (sublattice). $S$ is called a lower rough ideal (sublattice) if $\psi_{\star}(S)$ is an ideal (sublattice). $S$ is called a rough ideal of $L$ if it is both an upper rough ideal and a lower rough ideal. 
4.4. Definition. Let $\psi$ be a congruence relation on $L$, then $\psi$ is called a complete congruence relation if $[a]_{\psi} \vee[b]_{\psi}=[a \vee b]_{\psi}$ and $[a]_{\psi} \wedge[b]_{\psi}=[a \wedge b]_{\psi}$ for all $a, b \in L$.

If $\psi$ is a complete soft congruence relation on $L$, we define the pair $(L, \psi)$ as complete soft congruence approximation space over $L$.

4.5. Proposition. Let $\psi$ be a soft congruence relation on a non-empty set L. If $A$ and $B$ are non-empty subsets of $L$, then

(1) $\psi_{\star}(A) \subseteq A \subseteq \psi^{\star}(A)$,

(2) $\psi^{\star}(A \cup B)=\psi^{\star}(A) \cup \psi^{\star}(B)$,

(3) $\psi_{\star}(A \cap B)=\psi_{\star}(A) \cap \psi_{\star}(B)$,

(4) $A \subseteq B \Rightarrow \psi^{\star}(A) \subseteq \psi^{\star}(B)$ and $\psi_{\star}(A) \subseteq \psi_{\star}(B)$

(5) $\psi_{\star}(A \cup B) \supseteq \psi_{\star}(A) \cup \psi_{\star}(B)$,

(6) $\psi^{\star}(A \cap B) \subseteq \psi^{\star}(A) \cap \psi^{\star}(B)$.

4.6. Proposition. Let $L$ be a lattice and $(L, \psi)$ be a complete soft congruence approximation space over $L$. If $A$ is a sublattice of $L$, then $A$ is an upper rough sublattice.

Proof. Let $a, b \in \psi^{\star}(A)$, then $[a]_{\psi} \cap A \neq \phi$ and $[b]_{\psi} \cap A \neq \phi$, so there exist $x$ and $y$ such that $x \in[a]_{\psi} \cap A$ and $y \in[b]_{\psi} \cap A$. So, $x, y \in A$ and since $A$ is a sublattice, hence $x \vee y \in A$. Again $x, y \in A$ and $\psi$ is a complete soft congruence relation on $L$, therefore $x \vee y \in[a]_{\psi} \vee[b]_{\psi}=[a \vee b]_{\psi}$. Therefore $x \vee y \in[a \vee b]_{\psi} \cap A$, so $a \vee b \in \psi^{\star}(A)$. Similarly we can prove $a \wedge b \in \psi^{\star}(A)$. So, the proposition is obvious.

4.7. Proposition. Let $L$ be a lattice and $(L, \psi)$ be a complete soft congruence approximation space over $L$. If $A$ is a sublattice of $L$, then $A$ is a lower rough sublattice of $L$ if it is non-empty.

Proof. Let $a, b \in \psi_{\star}(A)$, then $[a]_{\psi} \subseteq A$ and $[b]_{\psi} \subseteq A$. But $\psi$ is a complete soft congruence relation on $L$, therefore $[a]_{\psi} \vee[b]_{\psi}=[a \vee b]_{\psi}$. Let $k$ be any element of $[a \vee b]_{\psi}$. So there exist $x \in[a]_{\psi}$ and $y \in[b]_{\psi}$ such that $k=x \vee y$. Now $x \in[a]_{\psi} \subseteq A$ and $y \in[b]_{\psi} \subseteq A$ i.e., $x, y \in A$, and since $A$ is a sublattice, $k=x \vee y \in A$, that is $k \in[a \vee b]_{\psi}$ imply $k \in A$. So $[a \vee b]_{\psi} \subseteq A$. Therefore $a \vee b \in \psi_{\star}(A)$. Similarly we can prove $a \wedge b \in \psi_{\star}(A)$. Hence the proof is completed.

4.8. Proposition. Let $\psi$ be a congruence relation on a lattice $L$. If $A$ and $B$ are ideals of $L$, then $\psi^{\star}(A \cap B)=\psi^{\star}(A) \cap \psi^{\star}(B)$.

Proof. Let $x \in \psi^{\star}(A) \cap \psi^{\star}(B)$. Then $[x]_{\psi} \cap A \neq \phi$ and $[x]_{\psi} \cap B \neq \phi$. Then there exist $y \in A$ and $z \in B$ such that $x \psi y$ and $x \psi z$ hold. So we can write $(x \vee x) \psi(y \vee z)$ that is $x \psi(y \vee z)$. Since $A$ and $B$ are ideals of $L$, we have $y \wedge z \in(A \cap B)$ and hence $[x]_{\psi} \cap(A \cap B) \neq \phi$ which implies $x \in \psi^{\star}(A \cap B)$ that is $\psi^{\star}(A) \cap \psi^{\star}(B) \subseteq \psi^{\star}(A \cap B)$. Also by Proposition $4.5, \psi^{\star}(A \cap B) \subseteq \psi^{\star}(A) \cap \psi^{\star}(B)$. Hence we have $\psi^{\star}(A \cap B)=$ $\psi^{\star}(A) \cap \psi^{\star}(B)$.

4.9. Proposition. Let $\psi$ be a complete soft congruence relation on a lattice $L$. If $A$ is an ideal of $L$, then $A$ is an upper rough sublattice of $L$.

Proof. If $A$ is an ideal of $L$, then $A$ is a sublattice of $L$ and then this proposition follows from the Proposition 4.6.

4.10. Proposition. Let $(F, A)$ and $(G, B)$ be soft congruence relations over $L$. Then $(H, C)=(F, A) \wedge(G, B)$ is soft congruence relation over $L$. 
Proof. Let $\left(a_{1}, b_{1}\right)$ and $\left(a_{2}, b_{2}\right) \in H(\alpha, \beta)$ for all $(\alpha, \beta) \in A \times B$. Then $\left(a_{1}, b_{1}\right)$ and $\left(a_{2}, b_{2}\right) \in F(\alpha)$, and $\left(a_{1}, b_{1}\right)$ and $\left(a_{2}, b_{2}\right) \in G(\beta)$. Since $(F, A)$ is congruence relation, $\left(a_{1} \wedge a_{2}, b_{1} \wedge b_{2}\right),\left(a_{1} \vee a_{2}, b_{1} \vee b_{2}\right) \in F(\alpha)$. Similarly, $\left(a_{1} \wedge a_{2}, b_{1} \wedge b_{2}\right),\left(a_{1} \vee a_{2}, b_{1} \vee b_{2}\right) \in G(\beta)$. Thus, $(H, C)$ is congruence relation over $L$.

4.11. Proposition. Let $\left\{\left(F, A_{i}\right): i \in I\right\}$ be a non-empty family of soft congruence relation over $L$. Then,

$$
\bigwedge\left\{\left(F, A_{i}\right): i \in I\right\}
$$

is soft congruence relation over $L$.

\section{Conclusions}

Soft congruence relation is a new kind of soft set relation. In this paper, we have established the soft congruence relation over lattice. Several properties of soft congruence relation have been studied. Approximations of subset of a lattice have been studied with respect to soft congruence relation. That is the roughness of a subset of lattices has been discussed using the soft set relation. We have also discussed the properties of lattice ideal with respect to the soft congruence relation. In addition to the above, we have concluded that the concept of the paper has opened a new platform for algebraic study.

As an extension of this work, one can concentrate on the following topics:

(1) Designing the soft lattice based on soft congruence relation.

(2) Formulating the fuzzy soft congruence relation.

(3) Defining the soft congruence relation of soft lattice.

\section{Acknowledgements}

The authors are very much thankful to the anonymous reviewer for his valuable suggestions and comments which helped us to improve the quality of the paper.

\section{References}

[1] Ali, M.I., Feng, F., Liu, X., Min, W. K. and Shabir, M. On some new operations in soft set theory, Computers and Mathematics with Applications 57, 1547-1553, 1999.

[2] Babitha, K.V. and Sunil, J.J. Soft set relations and functions, Computers and Mathematics with Applications 60, 1840-1849, 2010.

[3] Babitha, K.V. and Sunil, J. J. Transitive closures and ordering on soft sets, Computers and Mathematics with Applications 62, 2235-2239, 2011.

[4] Bera, S. and Roy, S.K. Rough modular lattice, Journal of Uncertain Systems 7, 289-293, 2013.

[5] Bozena, K. Soft set approach to the subjective assessment of sound quality, in: IEEE Conferences 669-674, 1998.

[6] Chen, D., Tsang, E.C.C., Yeung, D.S. and Wang, X. The parameterization reduction of soft sets and its applications, Computers and Mathematics with Applications 49, 757-763, 2005.

[7] Çağman, N., and Enginoğlu, S. Soft set theory and uni-int decision making, European Journal of Operational Research 207, 848 - 855, 2010.

[8] Estaji, A.A., Hooshmandasl, M.R. and Davvaz,B. Rough set theory applied to lattice theory, Information Sciences 200, 108-122, 2012.

[9] Feng, F., Ali, M. I. and Shabir, M. Soft relations applied to semi groups, Filomat 27(7), 1183-1196, 2013.

[10] Iwinski, T.B. Algebraic approach to rough sets, Bulletin of the Polish Academy of Sciences, Mathematics 35, 673- 683, 1987.

[11] Järvinen, J. Lattice theory for rough sets, Lecture Notes in Computer Science 4374, 400-498, 2007.

[12] Jang, Y., Tang, Y., Chen, Q., Wang, J., and Tang, S. Extending soft sets with description logics, Computers and Mathematics with Applications 59, 2087-2096, 2010. 
[13] Liao, Z., Wu, L. and Hu, M. Rough lattice, IEEE International Conference on Granular Computing 716-719, 2010.

[14] Molodtsov, D. Soft set theory- first results, Computers and Mathematics with Applications 37, 19-31, 1999.

[15] Maji, P.K., Biswas, R. and Roy, A.R. Soft set theory, Computers and Mathematics with Applications 45, 555-562, 2003.

[16] Milind, M.M., Sengupta, S. and Ray, A.K. Texture classification using a novel soft set theory based classification algorithm, Springer, Berlin, Heidelberg 246-254, 2006.

[17] Park, J.H., Kim, O.H. and Kwun, Y.C. Some properties of soft set relations, Computers and Mathematics with Applications 63, 1079-1088, 2012.

[18] Pawlak, Z. Rough Sets, International Journal of Computer and Information Sciences 11(5), 341-356, 1982.

[19] Pawlak, Z. Rough sets theoretical aspects of reasoning about data, Academic Publisher 1991.

[20] Roy, S.K. and Bera, S. Soft rough lattice, Kragujevac Journal of Mathematics 39, 13-20, 2015.

[21] Roy, S.K. and Bera, S. Approximation of rough soft set and its application to lattice, Fuzzy Information and Engineering 7, 379-387, 2015.

[22] Roy, S.K. and Bera, S. Soft rough approach to lattice-ideal, The Journal of Fuzzy Mathematics 24, 49-55, 2016.

[23] Roy, S.K. and Bera, S. Distributive lattice: a rough set approach, Malaya Journal of Matematik 2, 273-276, 2014.

[24] Rana, D. and Roy, S.K. Concept lattice: a rough set approach, Malaya Journal of Matematik 3(1), 14-22, 2015.

[25] Rana, D. and Roy, S.K. Rough lattice over Boolean algebra, Journal of New Theory 2, 63-68, 2015.

[26] Rana, D. and Roy, S.K. Homomorphism in rough lattice, Journal of New Theory 5, 19-25, 2015 .

[27] Rana, D. and Roy, S.K. Lattice of rough intervals, Journal of New Results in Science 2, 39-46, 2013.

[28] Xiao, Q.M., and Zhang, Z.L. Rough prime ideals and rough fuzzy prime ideals in semigroups, Information Sciences 176, 725-733, 2006.

[29] Rana, D and Roy, S.K. Rough set approach on lattice, Journal of Uncertain Systems 5 , 72-80, 2011.

[30] Rasouli, S., and Davvaz, B. Roughness in MV-algebras, Information Sciences 180, 737-747, 2010.

[31] Zadeh, L.A. Fuzzy sets, Information and Control 8, 338-353, 1965. 